\title{
Effects of acupuncture to treat fibromyalgia: A preliminary randomised controlled trial
}

\author{
Kazunori Itoh*, Hiroshi Kitakoji
}

\begin{abstract}
Background: Acupuncture is often used to treat fibromyalgia (FM), but it remains unclear whether acupuncture is effective. This study aims to evaluate the effects of acupuncture on pain and quality of life (QoL) in FM patients.

Methods: Sixteen patients (13 women and 3 men aged 25-63 years) suffering from FM were randomised into two groups: group A $(n=8)$ received five acupuncture treatments after the fifth week and group B received ten acupuncture treatments. Outcome measures used in this study were pain intensity (visual analogue scale, VAS) and the fibromyalgia impact questionnaire (FIQ).

Results: After the fifth week, pain intensity $(U=25.0 ; P=0.022)$ in group $B$ decreased and $Q$ Q $~(U=24.5$; $P=0.026)$ improved compared to group $A$.

Conclusion: The present study suggests that acupuncture treatment is effective to relieve pain for FM patients in terms of QoL and FIQ.
\end{abstract}

\section{Background}

Fibromyalgia (FM) is a condition of unknown cause; it is characterized by widespread musculoskeltal pain with symptoms including stiffness, fatigue, sleep disturbance and functional impairment $[1,2]$. According to the American College of Rheumatology criteria, an FM patient must have chronic widespread pain and at lest 11 out of 18 tender points on examination [1]. Affecting $2-4 \%$ of the populations in industrialized countries, FM is the second most common rheumatologic disorder in the world $[3,4]$. A wide range of treatment methods are currently in use such as medications, physical methods and manual treatments $[5,6]$. Complementary and alternative medicine (CAM) is commonly used to treat FM patients [7]. Ninety-one per cent (91\%) FM patients used CAM [8] and significantly more FM patients used CAM compared to patients of other rheumatologic diseases [9]. One of the most commonly used forms of CAM is acupuncture (annual utilization percentages, from $4.8-6.7 \%$ of lifetime experiences to $19.4-26.7 \%$ in Japan) [10]. Although acupuncture has been used for pain relief for a long time in China and around the world, studies on the efficacy of acupuncture on FM

\footnotetext{
* Correspondence: k_itoh@meiji-u.ac.jp

Department of Clinical Acupuncture and Moxibustion, Meiji University of Integrative Medicine, Hiyoshi-cho, Nantan, Kyoto 629-0392, Japan
}

provided mixed results $[1,11]$. Most studies which indicated beneficial effect of acupuncture treatment for FM were uncontrolled case series $[1,11,12]$; only a few supported that acupuncture was effective [1,13-16]. A recent systematic review found no evidence to show beneficial effects of acupuncture to treat FM compared with placebo [1]. Although efficacy of acupuncture have been assessed with various controls such as no-treatment controls [17], non-penetration needling [18], minimal acupuncture $[19,20]$ and mock transcutaneous electrical nerve stimulation (TENS) [21,22], most studies on the efficacy of acupuncture for FM were uncontrolled case series that utilized electroacupuncture treatment $[1,11]$, which make the setting of controls difficult because it involves perceptible current [23]. One of the alternative ideas which enable evaluation of the efficacy of acupuncture acceptable may be utilization of standard medication as a control condition. The present study aims to determine the efficacy of acupuncture in the symptomatic treatment for FM in comparison to the commonly-used medications.

\section{Methods \\ Patients}

Participants in the present study were 16 patients who were diagnosed with FM by specialists in respective 
hospitals and visited Acupuncture and Moxibustion Center, Meiji University of Integrative Medicine (Kyoto, Japan), seeking ways in relieving symptoms. Inclusion criteria were: (1) having met the American College of Rheumatology criteria for the diagnosis of FM for at least one year; (2) widespread pain for six months or more; (3) normal neurological examination findings of nerve function, including deep tendon reflexes, voluntary muscle action and sensory function; and (4) failure to respond to medications prescribed by FM specialists. Exclusion criteria were: (1) sufficient knowledge of acupuncture which may prevent blinding (e.g. having received acupuncture previously); (2) known bleeding diathesis; (3) having autoimmune or inflammatory diseases; (4) participation in other clinical trials; (5) pregnancy or lactation; or (6) receiving disability payments or involved in litigation related to FM. However, patients receiving FM medications were included if there had been no change in their medications for one month or longer prior to recruitment.

Patients having signed a written informed consent were recruited and randomly assigned with a computerised randomisation program (SAMPSIZE V2.0, Blackwell Sience Ltd, UK, permutated block randomization) to either group A where patients received acupuncture treatment followed by a control period or group B where they received continuous acupuncture. This study was approved by the Ethics Committee of Meiji University of Integrative Medicine.

The two groups received a total of five (group A) or ten (group B) acupuncture treatments once a week. Each session lasted for 30 minutes. Group A (control) received a total of five acupuncture treatments after the control period of five weeks. Patients in group A received clinical examinations once or twice a week for over five weeks by a FM specialist prior to the intervention period whereas those in group $B$ received a total of 10 acupuncture treatments (Figure 1).

\section{Blinding}

Outcome measures were performed by an independent investigator who was not informed of the treatment sequence or the treatment the patient had received prior to each measurement.

\section{Intervention}

Electroacupuncture and trigger point acupuncture were used to treat the patients. Patients received 15 minutes of electroacupuncture and then 15 minutes of trigger point acupuncture. Four pairs of electrodes were placed on the forearms and lower legs of the patients who were connected to a pulse generator (OhuPulser LFP4000A, Zen Lryoki Corp, Japan). The current was rectangular with a biphasic top and a frequency of $4 \mathrm{~Hz}$. Intensity of the current was set between the perception and pain thresholds inducing a visible muscular contraction. Disposable stainless steel needles $(0.2 \mathrm{~mm} \times$ $40 \mathrm{~mm}$, Seirin, Japan) were inserted into the skin over the point to a depth of 5-20 mm. Depth of insertion was determined according to the patient's needling sensation of the specific site. Both sides of four common acupuncture points were used for patients receiving electroacupuncture.

Up to ten additional sites were chosen according to the patient's symptoms and pain pattern as well as the empirical choice of trigger point in pain treatment. Disposable stainless steel needle $(0.2 \mathrm{~mm} \times 40 \mathrm{~mm}$, Seirin, Japan) was inserted into the skin over the trigger point to a depth of $10-20 \mathrm{~mm}$, appropriate to the muscle (Table 1), attempting to elicit a local muscle twitch

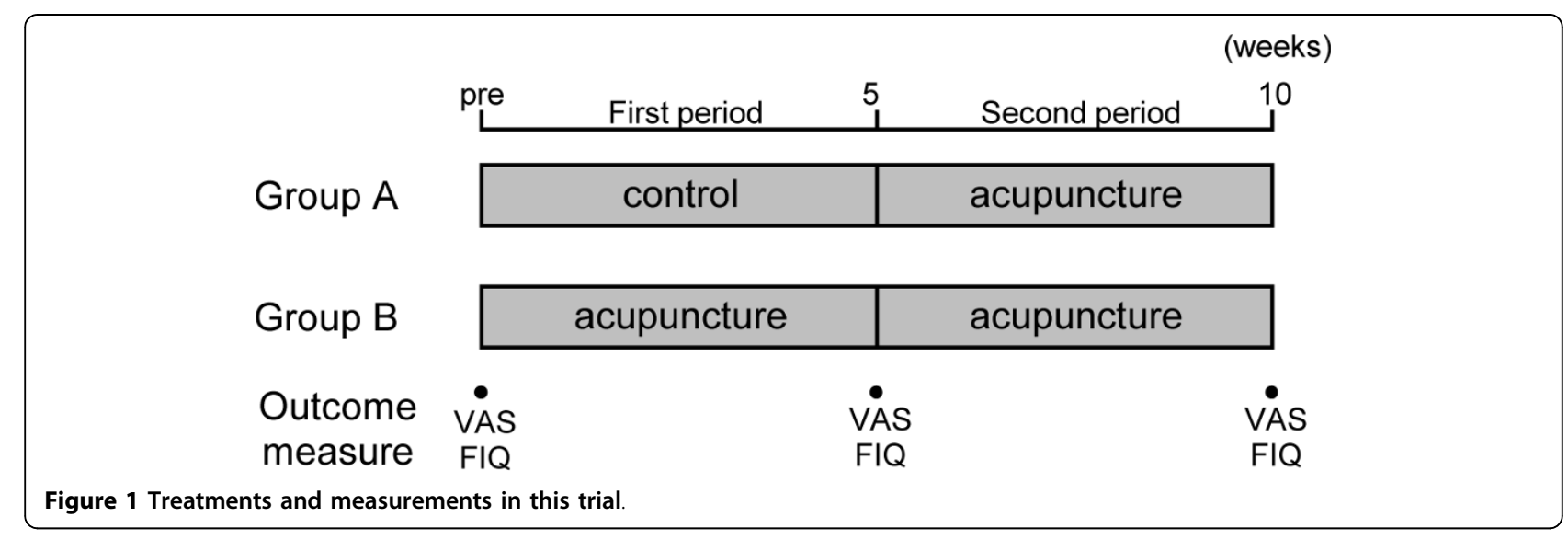


Table 1 Muscles treated in the two trigger point acupuncture groups

\begin{tabular}{lcc}
\hline Muscle & Group A & Group B \\
\hline Sternocleidomastoideus & $5^{*}$ & 5 \\
Trapezius & 6 & 5 \\
Pectoralis major & 5 & 5 \\
Quadratus lumborum & 4 & 4 \\
Erector spinae & 6 & 5 \\
Gluteus medius & 5 & 4 \\
Hamstrings & 4 & 3 \\
Other & 5 & 4
\end{tabular}

*number of the patients of which the indicated muscle was stimulated with the acupuncture needle (summation of these numbers is not equal to the total number of the participants because of duplicated counting)

response using the 'sparrow pecking' technique. After the local twitch response or a reasonable attempt, the needle was retained for ten more minutes.

The acupuncture was performed by an acupuncturist who had four years of acupuncture training and clinical experience of three or ten years.

\section{Evaluation}

Primary outcome measures included pain intensity quantified with a $10 \mathrm{~cm}$ visual analogue scale (VAS) and pain disability measured with the fibromyalgia impact questionnaire (FIQ) [24] on physical function, work, wellbeing. FIQ includes VAS for pain, sleep, fatigue, stiffness, anxiety and depression. Total score ranges between 0-100 with a higher score indicating a negative impact.

The VAS and FIQ measures were completed by the patients immediately before each treatment and analysed immediately before the first treatment (pre), five and ten weeks after the first treatment.

\section{Statistical analysis}

Outcome measures are reported as medians and interquartile ranges. Mann-Whitney $U$ test was used to analyse differences between groups at each time period. A difference was considered statistically significant when the $P$ value was less than 0.05 . The success of blinding was analysed by Fisher's exact test. Statistical package SYSTAT 12 (SYSTAT Software Inc, USA) was used to perform all the statistical analyses in this study.

\section{Results}

\section{Patient characteristics}

Sixteen participants (13 women and 3 men aged 2563 years) were randomised (Figure 2). No statistically significant differences in terms of age, pain duration, VAS and treatments received were found between the two groups at baseline (Table 2).

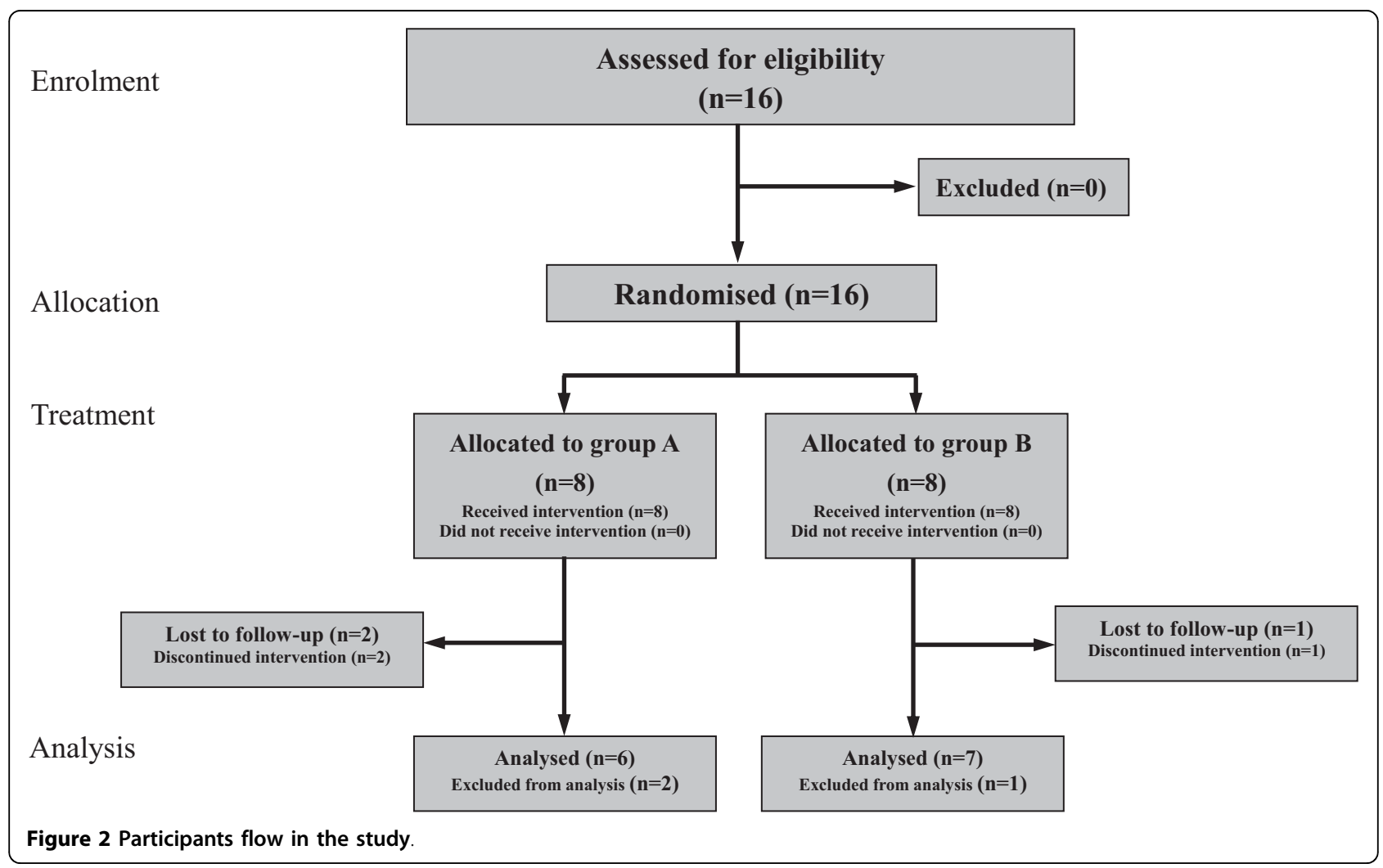


Table 2 Characteristics of patients in the three groups

\begin{tabular}{|c|c|c|}
\hline & Group A & Group B \\
\hline Sample size & 7 & 6 \\
\hline Age & $45.7(15.2)$ & $47.3(13.3)$ \\
\hline Pain duration (y) & $3.9(3.9)$ & $4.4(2.3)$ \\
\hline VAS $(\mathrm{mm})$ & $74.2(8.4)$ & $77.9(10.1)$ \\
\hline FIQ & $64.3(6.4)$ & $66.3(11.0)$ \\
\hline \multicolumn{3}{|c|}{ Treatments received: } \\
\hline Amitriptylin & 4 & 4 \\
\hline SSRIs & 2 & 3 \\
\hline SNRIs & 2 & 1 \\
\hline
\end{tabular}

Data are expressed as mean (standard deviation)

Two patients in group A and one patient in group B dropped out due to a lack of response to treatments. The dropout rates were not significantly different between the groups $(P=0.52$, Fisher's exact test). Analyses were performed on the 13 patients who did complete the study.

\section{VAS scores}

While the median VAS score in group A remained unchanged, those in group B decreased by the fifth week of treatment (Figure 3). However, the VAS score in group A began to decrease after the fifth week when the patients in this group also started receiving acupuncture treatment. There was a significant difference in the VAS scores between groups A and B at the fifth week $(U=25.0, P=0.022)$, whereas there was no significant difference between the groups at baseline $(U=5.0$, $P=0.566)$ and at the tenth week $(U=13.0, P=0.252)$ (Figure 3 and Table 3).

\section{QoL impairment}

While the FIQ score decreased after acupuncture treatments in group B for five weeks, those in group B remained unchanged. However, FIQ scores in both groups A and B decreased after the fifth week when patients in both groups received acupuncture. There was a significant difference between groups at the fifth

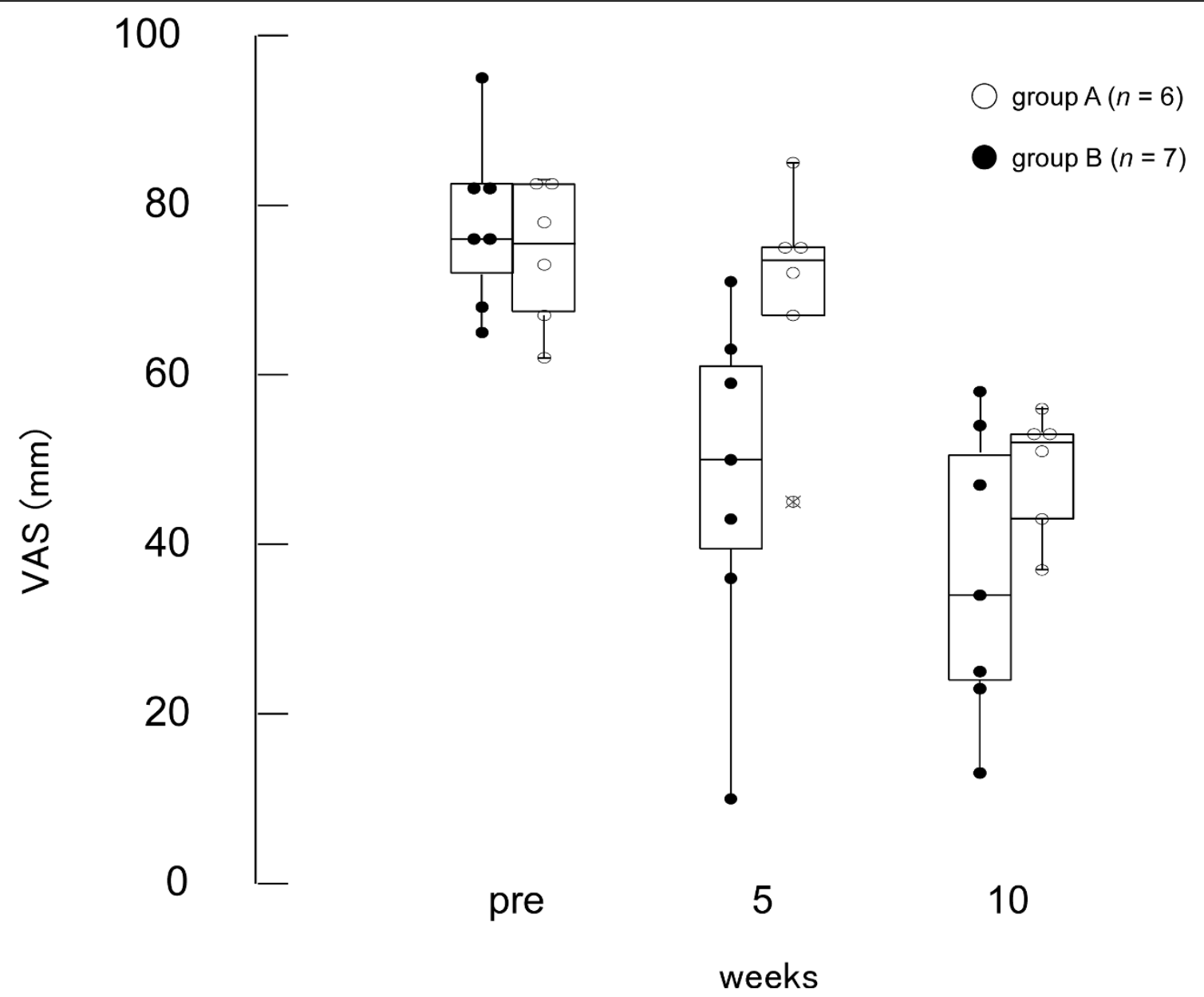

Figure 3 Effects of acupuncture on VAS score. There was a significant difference between group $A$ and $B(U=25.0, P=0.022)$, while no significant difference between groups at baseline $(U=5.0, P=0.566)$ and tenth week $(U=13.0, P=0.252)$. 
Table 3 Pain intensity VAS scores

\begin{tabular}{ccc}
\hline Week & Group A & Group B \\
\hline Pre & $75.5(68.5-81.0)$ & $77.8(72.0-82.5)$ \\
5 & $73.5(68.3-75.0)$ & $47.4(39.5-61.0)^{*}$ \\
10 & $52.0(43.0-53.0)$ & $36.3(24.0-50.5)$ \\
\hline
\end{tabular}

Data are medians (interquartile ranges). ${ }^{*} P<0.05$.

week $(U=24.5, P=0.026)$, whereas there was no difference between the groups at baseline $(U=5.5$, $P=0.616)$ and at the tenth week $(U=9.0, P=0.086)$ (Figure 4 and Table 4).

\section{Discussion}

The present study demonstrated a statistically significant difference between the acupuncture and active control (standard medication) groups five weeks after the first treatment. Moreover, the additionally performed acupuncture treatment after the fifth week in the control group resulted in the further reduction of the FM symptoms. These results suggest that acupuncture treatment is capable of giving additional improvement to the standard medication in the treatment of FM.

The importance of randomised controlled trials (RCT) to study the placebo effect of acupuncture was debated $[25,26]$. Acupuncture RCTs with various control methods such as no-treatment controls [17], nonpenetration needling [18], minimal acupuncture $[19,20]$ and mock transcutaneous electrical nerve stimulation (TENS) $[21,22]$ have been carried out. However, positive results were often obtained in studies with a nonacupuncture control [26,27], whereas negative results tended to be from those with sham acupuncture or mock TENS $[28,29]$.

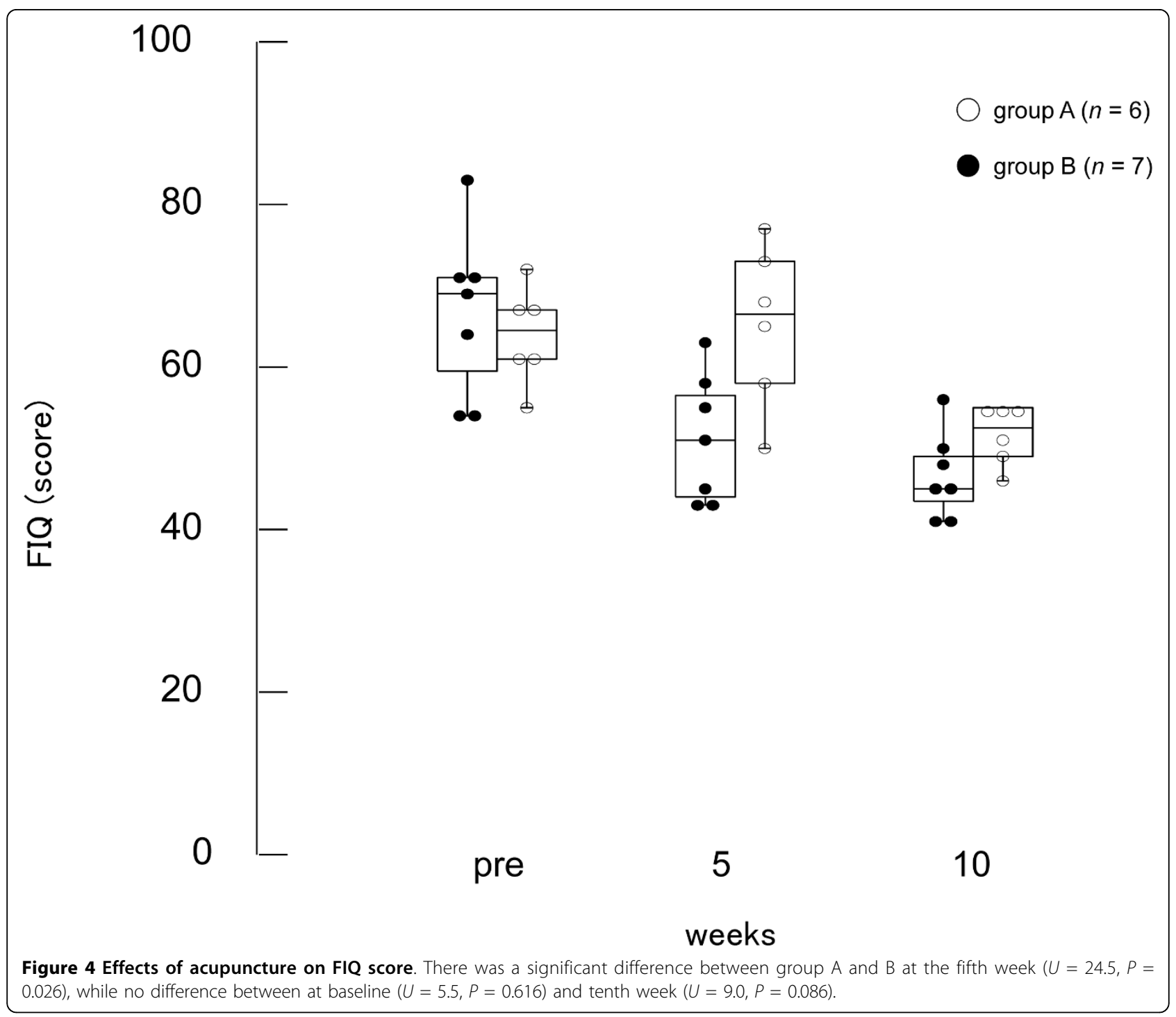


Table 4 Fibromyalgia Impact Questionnaire scores

\begin{tabular}{ccc}
\hline Week & Group A & Group B \\
\hline Pre & $64.5(61.3-67.0)$ & $66.7(59.5-71.0)$ \\
5 & $66.5(59.8-71.8)$ & $51.1(44.0-56.5)^{*}$ \\
10 & $52.5(49.5-54.8)$ & $46.7(43.5-49.0)$ \\
\hline
\end{tabular}

Data are medians (interquartile ranges). ${ }^{*} P<0.05$.

Moreover, most studies on the efficacy of acupuncture to treat FM were uncontrolled case series with electroacupuncture treatment $[1,11]$. As electroacupuncture involves perceptible current, sham acupuncture as control may not work for electroacupuncture. In fact, Targino et al. reported that addition of acupuncture to usual treatment for FM was beneficial for pain and QoL [16]. Our study also demonstrated that utilization of standard treatment as a control may be an ideal method to evaluate efficacy of acupuncture on FM. Although there were three patients who could not complete the study protocol, we consider the number had not much impact on the results because the numbers of withdrawal were similar between groups ( 2 out of 8 in group A and 1 out of 8 in group B). One of the limitations of the present study is relatively small sample size. Larger scale studies are required in the future.

\section{Conclusion}

The present study suggests that acupuncture treatment is effective to relieve pain for FM patients in terms of QoL and FIQ. Further larger scale clinical trials are warranted to confirm the findings of this study.

\section{Abbreviations}

RCT: randomised clinical trial; FM: Fibromyalgia; QoL: Quality of Life; VAS: visual analogue scale; FIQ: Fibromyalgia Impact Questionnaire; CAM: Complementary and alternative medicine; ACR: American College of Rheumatology; TENS: transcutaneous electrical nerve stimulation

\section{Acknowledgements}

The authors wish to thank Dr Y Katsumi and Dr N Ishizaki for his constructive comments on the manuscript and $\mathrm{H}$ Ochi and $\mathrm{K}$ Ashihara for their assistance during this study.

\section{Authors' contributions}

$\mathrm{KI}$ designed the clinical study, acupuncture and wrote the manuscript. HK designed and performed the statistical data analyses. Both authors read and approved the final version of the manuscript.

\section{Competing interests}

The authors declare that they have no competing interests.

Received: 12 May 2009 Accepted: 23 March 2010

Published: 23 March 2010

\section{References}

1. Mayhew E, Ernst E: Acupuncture for fibromyalgia - a systematic review of randomized clinical trials. Rheumatology 2007, 46:801-804.

2. Wolfe F, Smythe HA, Yunus MB, Bennett RM, Bombardier C, Goldenberg DL, Tugwell P, Campbell SM, Abeles M, Clark P, Fam AG, Farber SJ, Fiectner JJ, Franklin CM, Gatter RA, Hamaty D, Lessard J, Lichtbroun AS, Masi AT,
Mccain GA, Reynolds J, Romano TJ, Russell IJ, Sheon RP: The American College of Rheumatology 1990 crireria for the classification of fibromyalgia. Report of the multicenter criteria committee. Arthritis Rheum 1990, 33:160-172.

3. Wolfe F, Ross K, Anderson J, Russell IJ, Hebert L: The prevalence and characteristics of fibromyalgia in the general population. Arthritis Rheum 1995, 38(1):19-28.

4. Jacobsen S, Bredkjaer SR: The prevalence of fibromyalgia and widespread chronic musculoskeletal pain in the general population. Scand J Rheumatol 1992, 21(5):261-263.

5. Abeles M, Solitar BM, Pillinger MH, Abeles AM: Update on fibromyalgia therapy. Am J Med 2008, 121(7):555-561.

6. Clauw DJ: Pharmacotherapy for patients with fibromyalgia. J Clin Psychiatry 2008, 69(Suppl 2):25-29.

7. Branco JC, Saraiva F, Cerinic MM, Zoppi M, Martin N, Taieb C, Myon E: Fibromyaigia syndrome: a European epidemiological survey. Ann Rheum Dis 2005, 64(Suppl 3):1784.

8. Pioro-Boisset M, Esdaile JM, Fitzcharles MA: Alternative medicine use in fibromyalgia syndrome. Arthritis Care Res 1996, 9(1):13-17.

9. Breuer GS, Orbach H, Elkayam O, Berkun Y, Paran D, Mates M, Nesher G: Perceived efficacy among patients of various methods of complementary alternative medicine for rheumatologic diseases. Clin Exp Rheumatol 2005, 23(5):693-696.

10. Ishizaki N, Yano T, Kawakita K: Public status and prevalence of acupuncture in Japan. Evid Based Complement Alternat Med 2008, doi:10.1093/ecam/nen037.

11. Berman BM, Ezzo J, Hadhazy V, Swyers JP: Is acupuncture effective in the treatment of fibromyalgia? J Fam Pract 1999, 48(3):213-218.

12. Singh $B B$, Khorsan $R$, Vinjammury SP: Influence of comorbidities on improvement of fibromyalgia symptoms when treated with acupuncture: a short report. Altern Ther Health Med 2008, 14(5):24-25.

13. Lewis PJ: Electroacupuncture in fibromyalgia. BMJ 1993, 306(6874):393.

14. Harris RE, Tian X, Williams DA, Tian TX, Cupps TR, Petzke F, Groner KH, Biswas $P$, Gracely RH, Clauw DJ: Treatment of fibromyalgia with formula acupuncture: investigation of needle placement, needle stimulation, and treatment frequency. J Altern Complement Med 2005, 11(4):663-671.

15. Assefi NP, Sherman K, Jacobsen C, Goldberg J, Smith WR, Buchwald D: A randomized clinical trial of acupuncture compared with sham acupuncture in fibromyalgia. Ann Intern Med 2005, 143(1):10-19.

16. Targino RA, Imamura M, Kaziyama HHS, Souza LPM, Hsing WT, Furlan AD, Imamura ST, Raymundo SAN: A randomized controlled trial of acupuncture added to usual treatment for fibromyalgia. J Rehabil Med 2008, 40:582-588.

17. Coan RM, Wong G, Coan PL: The acupuncture treatment of neck pain: a randomized controlled study. Am J Chin Med 1982, 9(4):326-332.

18. Johansson A, Wenneberg B, Wagersten C, Haraldson T: Acupuncture in treatment of facial muscular pain. Acta Odontol Scand 1991, 49(3):153-158.

19. Leibing E, Leonhardt U, Koster G, Goerlitz A, Rosenfeldt JA, Hilgers R, Ramadori G: Acupuncture treatment of chronic low-back pain - a randomised, blinded, placebo-controlled trial with nine-month followup. Pain 2002, 96(1-2):189-196.

20. Harris RE, Zubieta JK, Scott DJ, Napadow V, Gracely RH, Clauw DJ: Traditional Chinese acupuncture and placedo (sham) acupuncture are differentiated by their effects on $\mu$-opioid receptors (MORs). Neuroimage 2009, 47(3):1077-1085.

21. Grant DJ, Bishop-Miller J, Winchester DM, Anderson M, Faulkner S: A randomised comparative trial of acupuncture versus transcutaneous electrical nerve stimulation for chronic back pain in the elderly. Pain 1999, 82(1):9-13.

22. Carlsson $\mathrm{CPO}$, Sjölund $\mathrm{BH}$ : Acupuncture for chronic low back pain: a randomised placebo-controlled study with long-term follow up. Clin J Pain 2001, 17(4):296-305.

23. Martin-Sanchez E, Torralba E, Diaz-Dominguez E, Barriga A, Martin JLR: Efficacy of acupuncture for the treatment of fibromyalgia: Systematic review and meta-analysis of randomized trials. Open Rheumatology J 2009, 3:25-29.

24. Rivera J, González T: The fibromyalgia impact questionnaire: a validated Spanish version to assess the health status in women with fibromyalgia. Clin Exp Rheumatol 2004, 22(5):554-560.

25. Vincent $C$, Lewith G: Placebo controls for acupuncture studies. J R Soc Med 1995, 88(4):199-202. 
26. Vincent CA, Richardson PH: The evaluation of therapeutic acupuncture: concepts and methods. Pain 1986, 24(1):1-13.

27. Birch S, Jamison RN: Controlled trial of Japanese acupuncture for chronic myofascial neck pain: assessment of specific and non-specific effects of treatment. Clin J Pain 1998, 14(3):248-255.

28. Moore ME, Berk SN: Acupuncture for chronic shoulder pain: an experimental study with attention the role of placebo and hypnotic susceptibility. Ann Intern Med 1976, 84(4):381-384.

29. Petrie JP, Hazleman BL: A controlled study of acupuncture in back pain. Br J Rheumatol 1986, 25:271-275.

doi:10.1186/1749-8546-5-11

Cite this article as: Itoh and Kitakoji: Effects of acupuncture to treat

fibromyalgia: A preliminary randomised controlled trial. Chinese Medicine 2010 5:11.

Submit your next manuscript to BioMed Central and take full advantage of:

- Convenient online submission

- Thorough peer review

- No space constraints or color figure charges

- Immediate publication on acceptance

- Inclusion in PubMed, CAS, Scopus and Google Scholar

- Research which is freely available for redistribution

Submit your manuscript at www.biomedcentral.com/submit 